ÉGYPTE monde arabe

\section{Égypte/Monde arabe}

6 | 2003

D'une intifâda l'autre. La Palestine au quotidien

\title{
Portrait d'un « nouveau réactionnaire » américain
}

A propos de The Hidden Hand, Middle East Fears of Conspiracy, Daniel Pipes, Macmillan Press, Londres, 1998 (1ère édition 1996)

\section{Anne-Claire Kerbœuf}

\section{(2) OpenEdition}

\section{Journals}

Édition électronique

URL : https://journals.openedition.org/ema/950

DOI : $10.4000 /$ ema.950

ISSN : 2090-7273

Éditeur

CEDEJ - Centre d'études et de documentation économiques juridiques et sociales

\section{Édition imprimée}

Date de publication : 31 décembre 2003

Pagination : 161-176

ISBN : 2-87027-993-0

ISSN : $1110-5097$

Référence électronique

Anne-Claire Kerbœuf, "Portrait d'un « nouveau réactionnaire » américain », Égypte/Monde arabe [En ligne], 6 | 2003, mis en ligne le 08 juillet 2008, consulté le 07 juillet 2022. URL : http://

journals.openedition.org/ema/950; DOI : https://doi.org/10.4000/ema.950

Ce document a été généré automatiquement le 7 juillet 2022

Tous droits réservés 


\title{
Portrait d'un « nouveau
} réactionnaire » américain

\author{
A propos de The Hidden Hand, Middle East Fears of Conspiracy, Daniel \\ Pipes, Macmillan Press, Londres, 1998 (1ère édition 1996)
}

\section{Anne-Claire Kerbœuf}

1 The Hidden Hand est un livre susceptible d'attirer l'attention, pour qui s'intéresse à l'histoire événementielle, au phénomène social de la rumeur ou à l'analyse des discours politiques au Moyen-Orient. Le recours à la rhétorique du complot y est en effet fréquent. Elle s'illustre dans le cas de l'Égypte par les interprétations immédiates auxquelles ont donné lieu l'attentat de Louxor en 1997 ou, plus récemment, le crash de l'avion d'Egypt Air en 1999. Ces interprétations accréditaient les Israéliens et/ou les Américains de la responsabilité de ces événements dramatiques qualifiés de "complots" sans que rien ne vienne fonder ces assertions. Or, sauf preuve du contraire, aucune étude approfondie n'a analysé cette pratique politique très usitée au cours $\mathrm{du} \mathrm{XX}^{\mathrm{e}}$ siècle. La récurrence de la rhétorique du complot, formulée le plus souvent par les médias, pose la question de la fabrication ou de la production de ces "théories». Qui en sont les producteurs? Quelles en sont les fins (dans le cadre de la politique intérieure et extérieure des pays concernés)? À qui s'adresse ces discours ? Quels sont les acteurs désignés du complot et pourquoi ? À quels imaginaire et réalités historiques s'accrochent-elles?

2 Autant de questions auxquelles le lecteur espère trouver quelque élément de réponse en ouvrant The Hidden Hand. Mais dès les premières pages, l'enthousiasme du lecteur averti est quelque peu entamé. D’entrée de jeu (préface et introduction), il apprend que la violence est «culturelle » dans cette partie du monde, l'auteur considérant en effet les théories du complot comme «une clé» pour comprendre «l'histoire de l'extrémisme et de l'instabilité politique de la région, sa culture de la violence, et son pauvre degré de modernisation» (p. 2). Armé de ce postulat et «avec toujours une abondance d'exemples à (sa) disponibilité », l'auteur prétend alors expliquer aux Occidentaux la complexité du «monde de la main cachée» (préface). Car le travail de Daniel Pipes n'est qu'une « enquête prélimiraire » qui « ne fait guère que documenter 
un phénomène et en offre une interprétation générale » (p. 6). Le lecteur aurait dès lors tort de ne pas prendre cet avertissement très au sérieux : il sera effectivement gavé d'exemples et d'assertions d'un haut degré de vulgarisation pour ne pas dire de désinformation et d'une pauvreté analytique affligeante (une «analyse » se profile à la IV e partie). «L'interprétation générale » de l'auteur, empreinte de multiples clichés sur le monde arabe', est en effet extrêmement subjective, bien que l'auteur se défende de tout parti pris («I have no covert purpose in writing on this topic », p. 7). Last but not least, Daniel Pipes, bien que concédant n'avoir pas les moyens d'analyser profondément le sujet, prétend aider «les Arabes et les Iraniens eux-mêmes (à) devenir plus conscients des effets odieux de la conspiration ». L'auteur se dit en effet porteur d'une "obligation morale", car selon lui et son maître à penser, Bernard Lewis, dont il reprend les propos, «ceux qui bénéficient de la démocratie libérale doivent exposer le linge sale des dictateurs, car nul autre ne le fera » (p. 7). Un devoir moral conféré aux Occidentaux, atténué une page plus loin par cette autre affirmation: «L'Occident rationnel, en somme, n'a aucune supériorité morale sur les autres civilisations, ni aucune raison d'être arrogant lorsqu'il s'agit du domaine de la paranoïa politique. Les lecteurs occidentaux feraient bien de se purger eux-mêmes de toute attitude supérieure. » (p. 9).

3 Le lecteur téméraire qui persistera dans sa volonté de lire The Hidden Hand, ses 17 chapitres et 404 pages, en apprendra finalement beaucoup plus sur Daniel Pipes luimême et sur la position de cet homme dans le champ intellectuel et politique américain, que sur le Moyen-Orient. Savoir ce que contient le livre d'un homme érigé en expert du Moyen-Orient par les médias américains et ce, particulièrement depuis le 11 septembre 2001, n'est pas inutile, le discours conservateur et réactionnaire qui s'y lit appartenant au courant de pensée dominant actuellement l'échiquier politique américain.

Les théories du complot : un « concept » fourre-tout

4 Dès l'introduction, le lecteur peut donc prendre la mesure de la rigueur scientifique de l'ouvrage de cet universitaire sortant de Harvard. Prétendre faire un livre d'un chapelet d'anecdotes «allègres», nullement contextualisées historiquement ni suivies d'aucune analyse, est assez édifiant. Du premier chapitre jusqu'à la conclusion, le lecteur se noie en effet dans une masse d'exemples qui se diluent dans leur nombre et perdent ainsi toute valeur illustrative. Rares sont les occasions où l'auteur explique pourquoi tel fait est selon lui une théorie du complot. Pipes n'argumente pas, il impose des exemples et la perception qu'il en a : au lecteur de se fier à son esprit critique. Cependant, certaines illustrations ne manqueront pas de laisser sceptique (et c'est un euphémisme) le lecteur ayant sa propre analyse critique.

5 Voici le premier exemple de "théorie du complot » présenté par Daniel Pipes: «En Égypte, en 1952, les Officiers libres, persuadés que les gouvernements britannique et américain étaient engagés dans une bataille pour l'hégémonie sur le Moyen-Orient, ont cherché à avoir de bonnes relations avec Washington » (chapitre 1, p. 14). Outre le fait qu'elle soit suivie d'absolument aucune explication, et à supposer qu'elle soit exacte', en quoi cette affirmation relève-t-elle de théories du complot? L'auteur ne ferait-il pas l'amalgame entre stratégie politique et rhétorique du complot? Là où il pourrait remplir d'un peu de substance politique ces prétendues «théories du complot » et parler plus rationnellement d'État policier, de propagande (surtout lorsqu'il évoque l'Iran ou des situations de guerre), d'action, de stratégie et même d'opinion politique, il 
persiste dans son ouvrage à utiliser cette notion très vague qui se révèle de plus en plus fourre-tout au fil des pages...

6 Dans l'unique chapitre (chapitre 3) construit avec un minimum de méthode, et concernant la notion de «Grand Israël » (qui dénonce les ambitions sionistes d'étendre le royaume juif du "Nil jusqu'à l'Euphrate»), l'auteur interroge l'origine de cette notion et sa "validité ». Dans un premier temps, il fait référence à la Bible, aux prédictions d'un diplomate britannique, aux spéculations des premiers sionistes au début du siècle et aux déclarations de Moshe Dayan en 1967 et de Menachem Begin en 1983 (p. 54). Ces quelques éléments d'explication sont ensuite réfutés d'un revers de la main par l'auteur qui, amputant l'histoire d'Israël, estime que les "déclarations de chrétiens européens et des premiers sionistes n'ont clairement qu'une importance limitée. Les prédictions externes peuvent difficilement être considérées comme sources légitimes pour le mouvement sioniste. » (p. 55). De même « concernant les déclarations belliqueuses attribuées à Jabotinsky, Begin et Dayan, elles sont toutes de seconde main et au mieux quelque peu douteuses. Selon toute vraisemblance, des ennemis les ont simplement inventées». Quant à la Bible, l'État d'Israël ne s'y réfère pas, les «révisionnistes» ne pensant d'ailleurs qu'en termes «stratégiques» et non « historiques ». (p. 57)

7 Enfin, la partialité de l'auteur, camouflée sous les centaines d'exemples que le lecteur même téméraire n'a pas toujours les moyens ni le temps d'aller vérifier, perce régulièrement dans des propos criants de désinformation et de subjectivité. Ainsi, Pipes illustre-t-il les « déclarations ahurissantes » que certains fonctionnaires occidentaux ou israéliens publieraient dès leur départ à la retraite, reproduisant et validant ainsi « les pires peurs du Moyen-Orient », par la biographie d'un ex-agent du Mossad, Victor Ostrovsky, qui écrit entre autres «âneries » dans By Way of Deception que «les forces israéliennes ont aidés les chrétiens à massacrer les Palestiniens à Sabra et Shatila. » (chapitre 15, p. 314). On voit ici que les dizaines de témoignages des survivants du massacre de civils palestiniens ne pèsent pas beaucoup dans la balance de Pipes.

8 Autre révélateur des grosses lacunes méthodologiques de l'auteur: les sources. Elles sont uniquement de seconde main (l'auteur affectionne beaucoup la presse et les romans), voire de troisième main (Pipes adore citer les citations des auteurs de sa bibliographie). Aucun entretien avec des personnalités politiques ou des gens du commun n'a été effectué, ce qui peut paraître assez lacunaire pour un travail concernant les sociétés du Moyen-Orient. Pipes affirme par exemple que le Pakistan est gangrené par les théories du complot. Il en veut pour preuve ce chiffre assez convaincant (jugez-en vous-même) tiré du Wall Street Journal : «100\% des Pakistanais... sont des théoriciens du complot » (introduction, p .6). L'auteur s'attarde davantage sur la société iranienne dont il semble avoir une plus grande " érudition » puisqu'il affirme que les théories du complot s'insinuent jusque dans l'intimité des couples: "Les complots de la CIA s'immiscent jusque dans les domaines les plus banals de la vie quotidienne. Ils sont derrière les querelles conjugales, au moins lorsque l'un des conjoints est américain.» (chapitre 6, p. 108). Le lecteur, curieux de savoir sur quoi l'auteur se base pour tenir de tels propos généralisés à toute la société iranienne, voire aux sociétés du Moyen-Orient dans leur ensemble, s'empresse alors sur la note de fin de chapitre et découvre la référence: Jamais sans ma fille, le best-seller américain internationalement connu et adapté au cinéma de Betty Mahmoody... Autre exemple démonstratif choisi par Pipes pour faire la preuve de la "peur hyperbolique des 
Britanniques, étendue de la demeure rurale la plus modeste au palais » (car aucun carré d'herbe ni de bitume ne saurait échapper à ce fléau), cette citation de citation : «Si une pauvre femme dans le Khurasan trouvait un matin un dangereux serpent dans sa cuisine, il est fort probable qu'elle pense que les Anglais l'aient planté là.» (p. 107)

9 À travers cette compilation d'exemples se dessine peu à peu le profil de l'homo-moyenoriental modelé par l'auteur. Dans ce qui est un « discours sur le discours ", Pipes n'a de cesse de souligner les contradictions des rhétoriques du complot, et ce faisant, le « style paranoïaque » et la stupidité des leaders (qui plus est, croient ce qu'ils disent ! chapitre 11 , p. 246) comme des gens du commun dans cette partie du monde. Le lecteur découvrira également non sans intérêt le "visage » que prend l'histoire sous les lectures méthamorphosiques de Pipes...

Nouvelles lectures de l'histoire coloniale

La lecture que fait Daniel Pipes de l'histoire de la colonisation est empreinte d'un puritanisme aigu : à lire l'auteur, les colonisés sont colonisés parce qu'ils veulent bien l'être, ou parce qu'ils sont assez bêtes pour l'être. Voyez plutôt.

11 S'agissant du premier grand moment nationaliste iranien écourté par les impératifs politiques et économiques britanniques, l'auteur nous apprend que la «suspicion envers les étrangers » du premier ministre Muhamad Musadaq, qui refusa en outre les «excellentes propositions» de la Banque mondiale pour gérer la nationalisation du pétrole', est la cause de son renversement (chapitre 1, p. 18). Plus loin, l'auteur ajoute, reprenant les propos d'un auteur américain, que «la frustration» engendrée par l'expérience nationaliste avortée sous Musadaq (1951-1953) «incita les Iraniens à trouver une explication quant à l'intelligence des Anglo-américains, explication plus réconfortante que celle qui les forcerait à examiner leur propre vulnérabilité.» (chapitre 14, p. 304)

12 Non contents de subir l'occupation, les colonisés sont responsables des conséquences de la colonisation et, en outre, de la date tardive de leur indépendance. L'auteur en veut pour preuve celle de l'Égypte qui fluctue entre 1922, 1936, 1952 et 1956... Ou bien encore le cas palestinien, fort explicite car encore actuel : « La première déclaration d'un État palestinien a pris place en 1948 et une seconde en 1988 ; d'autres devraient venir....» "Une telle ambiguïté », estime l'auteur, qui cite Bernard Lewis à l'envi, « a produit [au Moyen-Orient] une génération d'hommes politiques plus aptes à demander des responsabilités qu'à les assumer, avec une tendance à fuir la réalité qui n'a pas encore complètement disparu. Cela se manifeste par une dépendance aux théories du complot. » (chapitre 16, p. 338)

13 Enfin, il est assez amusant de voir l'auteur contourner tout au long de cet ouvrage, sans jamais la nommer, une réalité historique qui paraît assez essentielle pour la compréhension de la production des théories du complot, aussi bien à cause de ses conséquences humaines que politiques: la création de l'État d'Israël en 1948 (le véritable «traumatisme » des Arabes). Or, à lire Pipes, l'histoire d'Israël semble s'être écrite sans les Israéliens, et surtout sans les Palestiniens dont on perd tout à fait la trace. Pour conclure son chapitre «Grand Israël » (chapitre 3) où l'auteur, on l'a vu, a expédié un peu rapidement les causes de cette théorie en faisant l'impasse sur quelques facteurs historiques d'importance (l'invasion du Sud Liban, de la Syrie, de l'Égypte), Pipes affirme que « ces craintes extravagantes de l'expansionnisme sioniste empêchent les gens du Moyen-Orient de considérer Israël comme un État avec des impératifs sécuritaires normaux » soit " un État comme un autre ». De plus, « en excitant la haine 
des Arabes contre Israël, cela (les théories du complot) a persuadé de nombreux Israéliens de s'accrocher aux territoires qu'ils ont gagnés en 1967 et de ne pas donner de chance aux traités de paix» (p. 69). Car, comme n'a de cesse de répéter l'auteur, Israël domine la région militairement et a le pouvoir de délimiter ses frontières. État de fait qui n'empêche nullement Pipes de faire un parallèle historique entre la position des juifs persécutés en Europe aux XVIII et XIXe siècles avec celle (impérialiste donc) des Israéliens aujourd'hui...

14 Enfin, après avoir illustré sur trois chapitres (6-7-8, Partie II) le fait que « Sionistes et Impérialistes » étaient les principaux acteurs dans les théories du complot au MoyenOrient, Pipes en arrive à cette conclusion (il s'agit des premières "réflexions " après 160 pages) : « La perception qu'ont les pays du Moyen-Orient de la place d'Israël dans le monde suggère que, malgré un siècle d'entreprise sioniste, les musulmans sont incapables de comprendre les relations de l'État juif avec l'Occident. En tant que musulmans, ils n'arrivent pas à comprendre la résonnance émotionnelle d'une Bible commune et d'une quantité de traits caractéristiques judéo-chrétiens. En tant que gens du Moyen-Orient, ils ne savent pas voir au-delà de la confrontation des nationalismes pour comprendre les intérêts partagés entre ces pays. En tant que citoyens d'États autoritaires, ils ne perçoivent pas l'importance des liens personnels, culturels et politiques entre hommes libres. » Outre la monstruosité du propos, on peut souligner ici les contradictions et contre-vérités : alors que plus haut, l'auteur affirme que l'État d'Israël ne s'appuie pas sur la Bible, il le qualifie ici d'État « juif ». Idem, alors que dans le chapitre 3 (p. 56) l'auteur rappelle que "dans le récit biblique, les "descendants" d'Abraham incluent non seulement les juifs par Isaac mais aussi leurs " cousins ", les Arabes par Ismaîl », il les exclut ici de l'histoire biblique (on notera que les termes arabes/peuples du Moyen-Orient/musulmans, ou juif/israélien, ou chrétien/ occidentaux sont interchangeables chez l'auteur). Enfin, il est notoire que juifs et chrétiens se sont aimés de tout temps, après tout Jésus était juif...

On arrive ici à propos pour mettre en exergue l'obsession religieuse de cet universitaire américain qui veut « éclairer » la lumière des Autres. Si Pipes, on l'a vu, n'emplit pas les théories du complot d'un peu de substance politique, il le fait volontiers de substance religieuse. À sa lecture insolite de l'histoire coloniale, l'auteur ajoute en effet une touche de religiosité. Pour Daniel Pipes, les « agents surnaturels » comme « Dieu, Satan, le destin et les forces occultes » sont des " forces motrices de l'histoire" avec lesquels il faut compter (chapitre 12, p. 257). Grâce à cette logique, on apprend que l'accès à l'indépendance est une revendication religieuse propre aux musulmans et qu'en conséquence les musulmans ont moins bien vécu la colonisation que les autres («With their religious imperative for independence, Muslims found colonialism even more degrading than did other peoples. » p. 302)

Lors d'un brossage de portrait pour le moins hétérodoxe de Jamâl al-Dîn al-Afghânî', dépeint comme un comploteur hors-pair qui aurait donné son nom au "syndrome » des comploteurs («the Afghani syndrom»), l'auteur en vient à reprocher au penseur pan-islamique et réformateur éclairé du XXe siècle, de ne pas être un bon musulman! (" there is reason indeed to believe that his image as a Muslim hid skeptical, not to say atheistic, attitudes ", chapitre 16, p. 346)

17 Enfin dans la quatrième et dernière partie intitulée "Why so many conspiracy Theories?", l'auteur écrit un chapitre (chapitre 14) sur "The Trauma of Modern Islam », suggérant ainsi que l'islam, "moderne» est le facteur de cette «mentalité 
paranoïaque » tout en expliquant que la cause principale est «le sens anachronique de supériorité des musulmans » et leur "dédain de l'Ouest à un moment où le respect aurait été plus approprié ». Après 1800, selon notre " historien », «l'arrogance d'une époque se mua en la mentalité conspiratrice d'une autre.» (p. 299)

Mais Pipes ne s'arrête pas là. Torpillant au passage la "dependencia theory " (dont s'inspire le courant antimondialisation), en la mettant d'emblée dans le panier des théories du complot - occidentales celles-là - l'auteur va jusqu'à regretter que «la suspicion millénaire [des musulmans] envers l'ennemi chrétien se traduise facilement en termes économiques. » (p. 317)

Portraits croisés

19 Pour parachever le portrait de l'homo moyen-oriental dans les derniers chapitres de son livre (parties III et IV, chapitres 11, 14, 17 et conclusion), Daniel Pipes recourt à la psychanalyse et à son vocabulaire "clinique", tentant ainsi de répondre à ces questions fondamentales : «Is Muslim by nature different from the Westerners? » (on notera la symétrie Muslim/Westerners) et «Can Westerners Help? »

Le lecteur très téméraire, qui à ce stade s'interrogera sur la discipline à laquelle pourraient bien relever l'ouvrage et son auteur, apprendra donc qu'il existe trois cas de paranoïaques cliniques: les "fanatiques ", les "cyniques » et les "énigmatiques » (chapitre 11, p. 241). Dans un joli parallèle avec les leaders nazis (Hitler, Goebbels, Rosenberg), Pipes classifie alors scientifiquement les cas cliniques du Moyen-Orient: "Les leaders arabes et iraniens se répartissent également entre ces trois archétypes. Khuminî tombe dans la catégorie des fanatiques, Sadâm Husayn dans celle des cyniques et Yâsir 'Arafât dans celle des énigmatiques.» (p. 242). Les Égyptiens peuvent se réjouir, leur président est épargné : «En Égypte, Husnî Mubârak offre un heureux exemple. Modéré dans le style, il s'implique peu dans les complots, accuse rarement les autres de complots, et en retour est rarement accusé de comploter»(conclusion, p. 377). Remarquons que le chef d'État égyptien étant un interlocuteur privilégié des ÉtatsUnis, cette constatation tombe à point nommé.

21 Le diagnostic étant fait, quels remèdes pour ces "paranoid patients "? Revêtu de sa blouse blanche, le docteur Pipes, qui a de la suite dans les idées, a ouvert avec soin et application The Encyclopedia of Human Behavior et trouvé ce conseil destiné à «ceux traitant cliniquement les patients paranoïaques »: "Trouvez un juste milieu entre défier franchement les illusions du patient et les renforcer en acquiessant" (conclusion, p. 376). Le Dr Pipes engagé dans la croisade contre la conspiration et muni désormais d'un «esprit constructif de compréhension" (p. 376), prodigue alors en cinq pages (ce qui est un peu léger pour un livre de 404 pages) quelques potions dont les mots clés sont « Teach », « Deny », « Refute », « Do not conspire » (p. 376-380).

Le lecteur, cependant, reste sur sa faim : quelles solutions en effet pour les maux qui minent les "structures politiques" (sic) de ces pays du Moyen-Orient, comme spécifiquement l'« hypocrisie » et «l'effet miroir » («mirror-imaging », chapitre 17)? Que faire contre la «qualité fluide » des gens du Moyen-Orient à mijoter des complots (chapitre 16, p. 340) ? À ces ultimes questions, point de réponses. À moins qu'il faille les trouver dans les phrases finales de ce brûlot: «Le problème finalement est moins lié aux actions des étrangers qu'à la situation et la psychologie des gens du MoyenOrient ». Les Occidentaux ne peuvent en définitive pas vraiment aider les Arabes qui doivent d'abord s'aider eux-même. 
i, on sera tenté d'affirmer, après la lecture intégrale de The Hidden Hand, que, inversement, Daniel Pipes ne peut pas vraiment aider les "Moyen-Orientaux », ni les Occidentaux d'ailleurs, et ferait bien de commencer par s'aider lui-même. En effet, au fil des pages, le lecteur constatera, non sans amusement, que l'auteur réunit toutes les caractéristiques du prototype du «théoricien du complot » tel que décrit par lui-même (chapitres 11 et 12). En voici quelques traits :

- «Submergé par ses propres émotions, le théoricien du complot ne les remarque qu’à peine ; et même si on le lui fait valoir, il n'y prête pas attention. Rarement prend-il de la distance avec lui-même ou mesure-t-il ses incohérences. » (p. 227)

- «Les théoriciens du complot semblent se contenter d'en savoir peu sur leurs ennemis. Ils recourent à un langage vague, attribuant des actes à des individus inconnus, usant des mots imprécis ou utilisant le mystérieux “ils". » (p. 253)

- «Quand ça l'arrange, le théoricien du complot utilise des preuves peu probantes. Tout type d'élément confirmant une information sert de preuve. Tout ce qui conforte son idée devient rapidement un fait établi.» (p. 254)

- «Procéder à une analyse classique, c'est d'abord rassembler des données puis construire des théories (...) les théoriciens du complot font l'inverse, commençant tout d'abord avec un paradigme, puis arrangeant les informations pour le soutenir. » (p. 251)

- «Connaissant déjà la conclusion, les théoriciens du complot débutent des recherches dans l'unique but de parvenir à convaincre les autres. » (p. 254)

- «Le théoricien du complot fait l'impasse sur le contexte historique. Il n'a jamais recours à l'analyse comparative ou à une perspective plus élargie. » (p. 257) Ne cherchez plus : la «main cachée » est celle de Daniel Pipes.

Daniel Pipes par lui-même

Après la lecture de La Main Cachée de Pipes, le lecteur piqué de curiosité et voulant en savoir davantage encore sur cet auteur (s'il ne le connaît déjà), se rendra sur le site-web de celui-ci (www.danielpipes.org). Il aura le privilège de voir apparaitre le visage sombre et ténébreux de cet illustre personnage, de prendre connaissance de sa biographie et de tous ses faits d'armes : interviews, articles aux titres assez évocateurs tels que "The New Anti-Semitism», «How many American Muslims? ", « Nightmare on Temple Mount ", "The gap between Civilization and Barbarism ». Et de découvrir, que s'il est inconnu ou presque en Europe, tel n'est pas le cas aux États-Unis. Diplômé de Havard, Daniel Pipes a enseigné à l'université (Havard, Chicago) et a été le viceprésident du Fulbright Board of Foreign Scholarships en 1992-1995. Cet universitaire au capital spécifique déficitaire, tenu à distance par ses collègues (pour les raisons que l'on a évoquées plus haut et au vu de la haine qu'il leur témoigne aujourd'hui) n'a pas tardé à trouver sa place dans le monde de la presse où il s'est forgé une réputation d'expert du Moyen-Orient (il est chroniqueur du Jerusalem Post et du New York Post et écrit dans de multiples journaux et magazines dont New York Times, The Wall Street Journal, Washington Post). Côté business, Pipes vend ses services "d'expert» à travers The Middle East Forum, une «organisation indépendante " créée en 1994, dont il est le président, et qui "promeut les intérêts américains à travers des publications, la recherche, le consulting, la diffusion médiatique et l'éducation publique ». On peut flairer ici les relations étroites de Pipes avec le pouvoir. C'est en effet un « intellectuel » du pouvoir accompli (et il ne s'en cache pas), défendant les "intérêts » de son pays, c'est-à-dire ceux de la droite conservatrice. M. Pipes, lit-on dans sa biographie, «a témoigné sous serment devant de nombreux comités du Congrès et a travaillé pour

Égypte/Monde arabe, 6 | 2003 
quatre campagnes présidentielles ». Et il sert aujourd'hui au sein de la «Special Task Force on Terrorism Technologie » du Département de la Défense...

Homme influent médiatiquement et politiquement, Daniel Pipes ne l'a cependant jamais été autant que depuis cette date fatidique qui restera dans l'histoire des ÉtatsUnis comme dans sa carrière professionnelle. Depuis le 11 septembre 2002, Pipes est considéré comme l'homme qui avait brandi la menace du péril vert (il a publié une dizaine de livres entre 1980 et 1998 sur le sujet) et qui n'a pas été écouté. "Contrairement aux autres experts du Moyen-Orient, Daniel Pipes n'a pas eu besoin de se réinventer ou de changer ses opinions après le 11 septembre. Tout comme les autres événements qui ont jalonné l'année dans le Moyen-Orient, cette date a montré la clairvoyance de ses analyses précédentes. À l'exception de Pipes, rares sont les experts qui n'ont pas été mis dans l'embarras par les événements ", écrit Robert Kaplan", dans un éloge de Militant Islam Reaches America", dernier livre que Pipes, fort de sa nouvelle légitimité et plus offensif que jamais, n'a pas manqué de publier en 2002. L'anéantissement des tours jumelles a en effet suscité une polémique jusque dans le milieu universitaire et de la recherche américains, où les spécialistes du Moyen-Orient sont accusés de n'avoir pas su prévoir l'attaque de Ben Laden... En position de force, Pipes et ses compères sont entrés dans une guerre ouverte contre les universitaires et prônent comme alternative une façon populiste de faire de la recherche. Dans un compte rendu du livre de Martin Kramer, Ivory Towers on Sand: The Failure of Middle Eastern Studies in America, véritable pamphlet anti-intellectualiste", Pipes renchérit sur le discrédit dont s'est couverte «cette guilde d'experts (qui) a plus de données que de sens commun ». Mais, providentiellement selon lui, « journalistes, analystes, et anciens hauts fonctionnaires ont comblé le vide. Leur nombre est restreint et les universitaires les traitent de "faux intellectuels" qui produisent des analyses "supercifielles et retorses", mais ils parlent le langage que les Américains comprennent, agissent en temps opportun et savent faire passer leur message ». En d'autres termes, pour Pipes et sa clique, le plus important c'est de vulgariser et non de comprendre".

Profitant du climat maccarthyste régnant depuis le 11 septembre, Pipes a saisi l'occasion de faire le ménage sur le campus, faisant ainsi d'une pierre trois coups. Il ne s'en prend pas seulement aux universitaires et chercheurs fidèles à une certaine rigueur éthique et scientifique, mais dénonce plus largement tous ceux qui n'épousent pas ses idées politiques, c'est-à-dire les gens de gauche et ceux opposés à la politique du gouvernement israélien d'Ariel Sharon (ceux-ci étant souvent ceux-là) qui tombent sous le coup de l'accusation banalisée d'" antisémitisme ». Dans un article publié dans le New York Post (25/06/02) et intitulé "Extremists on Campus », notre hérault des idées ultra-conservatrices de l'actuel gouvernement américain et son collègue Jonathan Schanzer, ont établi une liste des professeurs accusés d'être des extrémistes de gauche et des antisémites pour leurs positions pro-palestiniennes. Cette liste est précédée d'une introduction assez éloquente: «Durant trois décennies, les extrémistes de la gauche ont dominé le milieu académique américain, vomissant des drôles de théories apparemment inoffensives sur la "déconstruction", le "post-modernisme", les "race, genre et classe", mais en vérité attaquant les États-Unis, leur gouvernement et leurs alliés. » D'où la nécessité, conclut Pipes, de « condamner et contenir le militantisme de gauche qui a trop souvent passé pour du savoir sur le Moyen-Orient» afin que les universités redeviennent des « institutions au discours civilisé ». 
27 Pipes ne s'est pas contenté de dénoncer, mais a mis au point un site-web (campuswatch.org) encourageant tout individu à dénoncer les professeurs et étudiants dans le milieu des études sur le Moyen-Orient qu'il jugerait intolérants. Cette initiative, qui a suscité une large polémique, a fait des petits. Une mère de famille a créé un site web (NoIndoctrination.org) appelant les étudiants, toutes disciplines confondues cette fois, à dénoncer les professeurs dont le chauvinisme serait déficient (pas assez " américains »). Il est également assez intéressant de jeter un coup d'œil sur le site CampusNonsense.com et de lire les éditoriaux et articles dans The National Review" de Stanley Kurtz qui fait la promotion de ces sites web car, selon lui, la délation publique est le dernier rempart contre la gauche...

28 Cette organisation de la délation et du fichage systématique des professeurs et étudiants pour leur opinion politique, acte digne de l'époque maccarthyste, est extrêmement grave à deux niveaux au moins: non seulement elle met en jeu l'autonomie du champ intellectuel américain, mais elle est plus largement révélatrice de la crise démocratique que traversent les États-Unis.

Où sont passés les intellectuels?

29 Il ne faudrait cependant pas croire que cette tendance réactionnaire est confinée aux seuls États-Unis. L'Europe, et notamment la France, sont également concernées. Les idées américaines s'importent en effet au pays des "Lumières" où la "visibilité médiatique " de certains "intellectuels » prévaut volontiers sur l'information et le savoir. Si ces intellectuels qui épousent sans retenue les idées de leurs confrères américains n'ont pas assez de poids pour ouvrir une guerre ouverte contre les «académiciens » - le champ de la recherche étant encore suffisamment autonome leurs prises de positions publiques ont de quoi faire réfléchir sur le rôle des intellectuels, mais aussi des universitaires et chercheurs. Dans un article éloquent et cinglant publié dans Le Monde diplomatique, Maurice Maschino"s'interroge : "Que leur est-il arrivé? Naguère en première ligne pour défendre avec courage, contre les pouvoirs et l'opinion publique, des causes désespérées (affaire Callas, affaire Dreyfus, indépendance de l'Algérie, paix au Vietnam, etc.), beaucoup d'intellectuels français d'Alain Finkelkraut à Jacques Julliard, de Philippe Sollers à André Glucksmann, de Luc Ferry à Pascal Bruckner et tant d'autres - semblent désormais s'aligner sur les thèses dominantes les plus frileuses et les plus conservatrices. Héraults de la mondialisation libérale, génuflecteurs transis des États-Unis, soutiens inconditionnels du général Sharon, obséquieux complimenteurs des grands patrons, adulateurs de tous les pouvoirs et principalement de celui des grands médias, ces « intellectuels » n'usurpentils pas leur fonction et ne trahissent-ils pas Voltaire, Hugo, Zola, Gide, Sartre, Foucault et Bourdieu?». Prenant à parti l'historien Pierre Nora qui sépare rigoureusement recherche et engagement politique (« D'ajouter de l'opinion à de l'opinion (...) à quoi cela servirait-il?»), Maschino pose la question du rôle des universitaires et des chercheurs: ne doivent-ils pas sortir de leur «tour d'ivoire» lorsque les « intellectuels » monopolisant la scène publique ne remplissent plus leur rôle - comme le fit Pierre Bourdieu dans ses « livres d'intervention »?

La « déviance » de ces intellectuels médiatiques et médiatisés s'est clairement illustrée après le 11 septembre 2001, événement qui vint entériner pour certains la thèse $\mathrm{du}$ " choc des civilisations " et renforcer de nombreux clichés sur le monde arabe devenu essentiellement « musulman », la religion venant interdire toute analyse politique. Loin de contrecarrer ou du moins nuancer les réactions extrêmes que provoquèrent le choc 
de l'attentat, nombre de ces personnalités « bien pensantes » ne firent qu'abonder dans le sens commun et jongler avec les amalgames. Le conflit israélo-palestinien en pâtit en premier lieu. La responsabilité des «intellectuels-journalistes» dans la circulation d'idées fausses et intolérantes sur la deuxième intifâda est d'ailleurs l'objet du petit livre de Joss Dray et Denis Sieffert, La guerre israélienne de l'information. Désinformation et fausses symétries dans le conflit israélo-palestinien. En procédant à l'analyse du discours des journalistes de la presse écrite et audiovisuelle sur ces deux dernières années, les auteurs montrent comment la plupart de leurs confrères ont joué la carte de la propagande israélienne en reprenant à leur compte les communications officielles israéliennes, sans lecture critique ni investigation préalables. Ils ont ainsi œuvré à la « déchéance médiatique » de Yâsir cArafât avec la construction du mythe selon lequel le leader palestinien aurait dit «non » à « l'offre généreuse » d'Israël et donc refusé la " paix » à Camp David en 2000". En outre, les deux auteurs démontrent de manière pertinente combien les intellectuels s'adonnant aux «tribunes libres» dans les hebdomadaires et quotidiens à grande diffusion ont contribué, et contribuent encore, à brouiller les cartes sur le conflit en substituant la psychologie ou la poésie à l'analyse politique. La pensée a-politique est en effet dans le vent lorsqu'il s'agit du monde arabe. «Les passions qui agitent le monde arabo-musulman se situent ailleurs, dans un autre registre que celui du politique» (p. 71) affirme Jacques Tarnero dans Le Monde en réponse à un article de l'ancien président du CRIF (Conseil représentatif des institutions juives de France), Théo Klein. L'« autre registre» dont nous parle ce " chercheur associé au CNRS " (c'est ainsi qu'il signe ses articles) est celui utilisé par Daniel Pipes, soit celui de la folie, des passions et d'un certain culturalisme historique....Ainsi les Palestiniens sont-ils les victimes et héritiers d'un monde arabomusulman qui « depuis la chute de Cordoue, la bataille de Lépante ou la dislocation de l'Empire ottoman [entretient] l'idée d'une revanche à prendre contre un ennemi protéiforme » (ibid., p. 72). À ce registre, le psychanalyste des tribunes de Libération, Daniel Sibony, ajoute celui de sa spécialité, la psychanalyse (domaine, on l'a vu, également prisé par Pipes). Un passage au scanner psychologique du conflit israélopalestinien donne le résultat suivant: «Cette guerre est celle du partage même, celui des esprits bien plus que celui de la terre. (...) En même temps, cette guerre semble nécessaire à chacune des deux parties pour élaborer son partage intérieur, sa ligne d'ombre et de lumière, son bord étrange et familier avec l'ennemi.» (ibid. p. 73). Et Sibony de conclure de manière beaucoup moins vague : «Car le drame des Palestiniens est qu'ils n'ont pas pris leur part à temps. [...] Ils voulaient tout, donc ils n'ont rien (et maintenant, ça coûte plus cher) » (ibid. p. 74). La haine « culturelle» des Arabes est une autre causalité du conflit: «La haine préexiste à ce dont elle prétend se nourrir ou s'originer ", écrit dans Le Monde Claude Lanzmann, directeur de la revue Les Temps modernes (ibid. p. 76). Propos partagé (toujours dans Le Monde) par Pascal Bruckner : « Ce qui motive le terrorisme, ce n'est pas telle ou telle erreur de l'Europe ou de l'Amérique - et Dieu sait si nous en avons commises -, c'est la haine pure et simple».

31 En décryptant les propos de ces intellectuels qui parlent au plus grand nombre, Joss Dray et Denis Sieffert mettent le doigt non seulement sur la désinformation généralisée concernant le conflit israélo-palestinien, mais aussi sur les idées racistes et intolérantes véhiculées par ces personnalités médiatiques qui placent l'appartenance communautariste avant l'appartenance républicaine, oubliant ainsi momentanément le système politique dont ils sont issus. Juifs et musulmans n'ont en effet pas le même traitement sous la plume de ces intellectuels qui se flattent par ailleurs d'être de bons 
républicains, convaincus de représenter "l'autorité intellectuelle» de la France. Comme le soulignent Sieffert et Dray, les traits caractéristiques évoqués ci-dessus ne se limitent pas aux seuls Palestiniens, ni aux Arabes de la région, mais s'étendent aux «Arabes » ou plutôt aux «musulmans » de France et d'Europe...Que le brûlot raciste de la journaliste italienne Oriana Fallaci" n'ait non seulement suscité que peu de réactions, mais ait fait l'objet d'éloges de la part du journaliste-intellectuel de France-Culture, Alain Finkielkraut, est pour le moins alarmant (ibid. p. 77). Il est certain que si les juifs avaient été la cible de l'auteur, c'est un tollé général qu'aurait soulevé cette publication qui trône ostensiblement sur les présentoirs des supermarchés alimentaires aussi bien que ceux du livre.

Dans un cadre plus général, la presse française tend à jouer la carte du communautarisme religieux en faisant par exemple la une, après le 11 septembre, d'actes de délinquance contre des synagogues (et non de ceux dirigés contre les mosquées, aussi nombreux sinon plus) ou en privilégiant l'amalgame entre antisémitisme et "anti-Sharonisme » ou " anti-violation-des-droits-de-l'homme ». Le parti pris, la pression politique et la logique commerciale ont néanmoins été poussés à leur comble par le quotidien Le Monde, qui publia le 18 janvier 2001, sur une pleine page, en encart publicitaire (!), un message du CRIF titré (en énormes caractères alors que la mention "publicité » est très discrète) : "Israël doit-il se suicider ?». Ledit "message ", écrit dans un langage compréhensible d'un enfant, se fait explicitement l'écho de la propagande israélienne du gouvernement Sharon:

«M. cArafât veut d'abord la création d'un État, qu'Israël est prêt à accepter sur la quasi-totalité des territoires palestiniens. Rien n'empêche M. 'Arafât d'accueillir autant de Palestiniens qu'il souhaite dans les frontières du futur État palestinien. Aujourd'hui, M. 'Arafât revendique, en plus, l'installation de 3,7 millions de Palestiniens à l'intérieur même d'Israël dont la population est à peine de 6 millions d'habitants. En réalité, M. cArafât veut deux États: l'un tout de suite; l'autre à terme, par submersion démographique d'Israël. Il demande donc à Israël de se suicider! Déjà, il libère des terroristes qui commettent des attentats. Le CRIF dénonce la politique de $\mathrm{M}$. cArafât qui risque de mener à la guerre. Le CRIF soutient la volonté et les efforts de paix d'Israël. »

Aux lecteurs choqués, qui protestèrent auprès du journal, le romancier et médiateur choisi, Robert Solé, confondant propagande, publicité et liberté d'expression, argumenta en ces termes: "Pour ce qui est de la publicité, les pro-Palestiniens disposent d'un droit de réponse implicite : un encart payant ne leur serait pas refusé. » (Le Monde, 27/01/01). L'histoire, comme la liberté d'expression, sortent quelque peu malmenées des feuilles françaises.

En adoptant de telles postures, les intellectuels et journalistes français concernés empêchent la compréhension des conflits et renforcent l'idée de communautarisme religieux et le sentiment d'intolérance qui en découle. Or, se faire le chantre de la désinformation, du discours manichéen, des fausses symétries, ne peut que favoriser «l'esprit de complot » décrit par Daniel Pipes. 


\section{BIBLIOGRAPHIE}

Dray J. et Sieffert D., 2002, La guerre israélienne de l'information. Désinformation et fausses symétries dans le conflit israélo-palestinien, Paris, La Découverte.

\section{NOTES}

1.L'auteur s'efforce, sans y parvenir, de parler des « Middle Easterners and Iranians » et non pas des «Arabs » dans leur ensemble, son étude épargnant - sans que l'on sache pourquoi - le Maghreb. Une nuance que l'auteur fait en réalité pour ne pas prêter le flanc aux accusations de « racisme » car généralisant ses idées à tous les Arabes. Ce qu'il fait quand même, on aura maintes occasions de s'en apercevoir.

2.Les États-Unis avaient compris que les Britanniques perdaient pied en Égypte et voyaient la déstabilisation du pays d'un mauvais œil ; ils entrèrent en contact avec les «Officiers libres».

3.Ils pèsent par contre dans la balance des juges belges qui ont entamé depuis l'année 2000 une procédure pénale à l'encontre des responsables libanais et israéliens (dont Ariel Sharon) du massacre.

4.Acte nationaliste par excellence dont s'est dans doute inspiré Gamal cAbd-al Nâsir pour la nationalisation du canal de Suez en 1956.

5.Quelques lignes plus haut, l'auteur souligne que la majorité des Américains soutient les aides économiques et militaires apportées par les États-Unis à Israël. Et l'auteur de s'exclamer : « Les théoriciens du complot ont tendance à ignorer ces détails dérangeants $»$.

6.Ce ne sont pas les seuls, l'auteur y incluant aussi des « natural agents » comme « human nature, heredity, the environment, culture, probability » et « the self ».... 7.On apprend qu'al-Afghânî a « son biographe» attitré en la personne de Elie Kedouri. 8.Daniel Pipes semble bien correspondre dans le champ universitaire à la catégorie de ces « agents ou (ces) entreprises qui sont les plus enclins à céder à la séduction des profits « externes » parce qu'ils sont moins riches en capital spécifique (scientifique, littéraire, etc.) et moins assurés des profits spécifiques que le champ leur garantit dans l'immédiat ou à terme plus ou moins éloigné. », Bourdieu, Pierre, 1996, Sur la télévision, Liber-Raisons d'Agir, Paris, p. 89

9.Dans « My Media Year. Some lessons »,11/09/02, The National Review, Pipes fait le compte de toutes les interventions qu'il a dû faire à la demande des médias après le 11 septembre.

10.Essayiste et un des mentors de la politique extérieure de George W. Bush. Pour en savoir plus sur la tendance politique de Kaplan et ses confrères, lire Philip S. Golub, "Les Dynamiques du désordre mondial. Tentation impériale », Le Monde Diplomatique, septembre 2002.

11.Pipes y compare notamment le danger islamiste au danger communiste qu'il faut éradiquer d'une manière ou d'une autre.

12.En réponse à $M$. Kramer, voir notamment l'article de Steve Heydemann (professeur de sciences politiques à l'université de Colombia et chercheur au Social Science Research Council), juillet 2002, « Middle East Studies after 9/11. Defending The 
Discipline ", Journal of Democracy, Vol. 13, n 3. Voir aussi la réponse de Joel Benein, président de la MESA (Middle East Studies Association), 30/09/02, « Who's Watching the Watchers ? », History News Network (www.hnn.us/articles/1001/htlm ): Benein y évoque les liens de Kramer avec la droite ultra-conservatrice américaine et la droite israélienne.

13. Ce mode de pensée est partagé par un certain nombre « d'intellectuels » français (voir plus bas).

14.Voir sur internet www.nationalreview.com notamment les articles suivants : 02/12/02 «Students fight back, 23/09/02 «Balancing the Academy », 16/05/02 « Antiamericanism in the Classroom ».

15. Maurice T. Maschino, « Intellectuels médiatiques. Les nouveaux réactionnaires », Le Monde Diplomatique, octobre 2002.

16.Thèse défendu par Samuel Huntington dans Le Choc des civilisations, Odile Jacob, Paris, 1996.

17.Cette désinformation et « mythification » des accords de Camp David est démontrée dans le détail par Tanya Reinhart dans Détruire la Palestine ou comment terminer la guerre de 1948, 2002, La Fabrique, Paris.

18.Fallaci, Oriana, La Rage et l'orgueil, 2002, Plon, Paris : l'auteur compare notamment les musulmans à des rats se reproduisant de manière prolifique ou les décrit comme passant « leur temps avec le derrière en l'air à prier cinq fois par jour ».

INDEX

Mots-clés : théorie du complot

\section{AUTEUR}

ANNE-CLAIRE KERBCEUF

Cedej 